ENTRE A LONGUE DURÉE E O SHORT PURÉE: ARQUEOLOGIAS PÓS-COLONIAIS DA HISTÓRIA INDÍGENA NA AMÉRICA DO NORTE COLONIAL

BETWEEN THE LONGUE DURÉE AND THE SHORT PURÉE: POSTCOLONIAL ARCHAEOLOGIES OF INDIGENOUS HISTORY IN COLONIAL NORTH AMERICA

Stephen W. Silliman

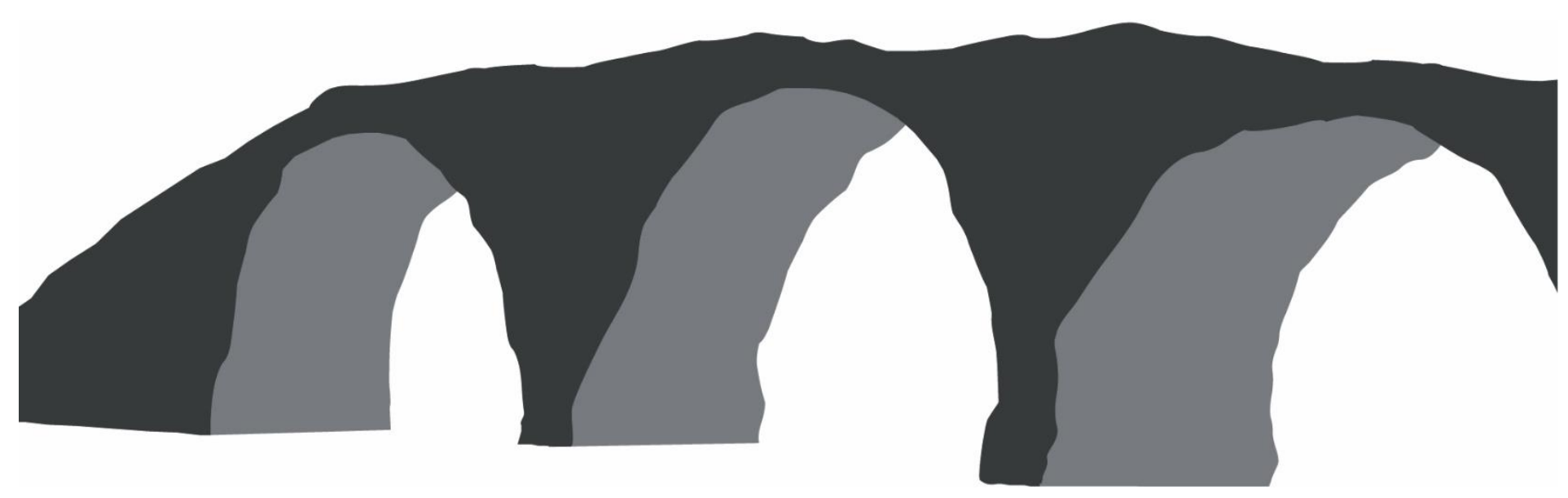




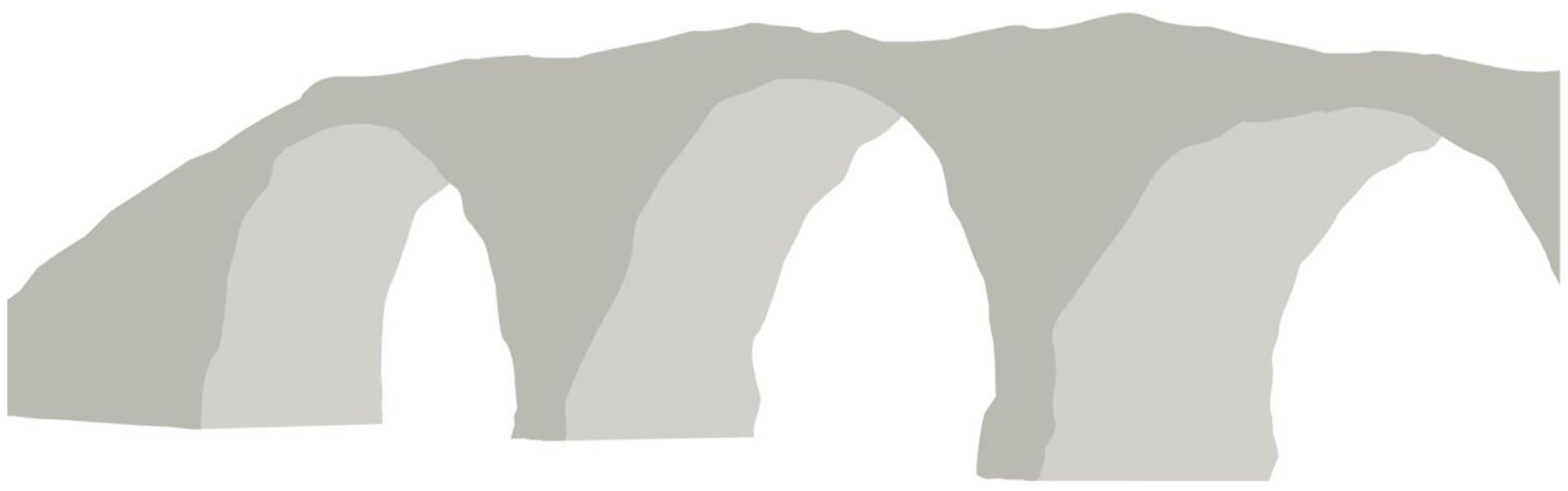

Publicação original: Silliman, Stephen W. 2012. Between the Longue Durée and the Short Purée: Postcolonial Archaeologies of Indigenous History in Colonial North America. In: Hart, S. M.; Oland, M; Frink, L. Decolonizing Indigenous Histories: Exploring "Prehistoric/Colonial" Transitions in Archaeology. Tucson (AZ), University of Arizona Press.

Reprinted by permission of the University of Arizona Press. 


\title{
ENTRE A LONGUE DURÉE E O SHORT PURÉE: ARQUEOLOGIAS PÓS-COLONIAIS DA HISTÓRIA INDÍGENA NA AMÉRICA DO NORTE COLONIAL ${ }^{1}$
}

\section{BETWEEN THE LONGUE DURÉE AND THE SHORT PURÉE: POSTCOLONIAL ARCHAEOLOGIES OF INDIGENOUS HISTORY IN COLONIAL NORTH AMERICA}

\author{
Stephen W. Silliman ${ }^{2}$
}

Arqueólogos que estudam culturas Indígenas no contexto do colonialismo Europeu estão frequentemente presos em um enigma de escala temporal. Como representar, traduzir e interpretar práticas e pessoas Indígenas de forma a não só respeitar as complexidades do mundo colonial e suas ações neste, mas também situar suas vidas no contexto de suas próprias histórias culturais únicas de curto e longo prazo? Capturar essa dualidade não tem sido fácil. Parte desse problema é que os arqueólogos não têm atendido completamente à chamada de Lightfoot (1995) a fim de conduzir verdadeiros estudos multiescala e diacrônicos sobre colonialismo e respostas Indígenas para as suas várias formas. Parte disso se relaciona às maneiras como conceitos arqueológicos, termos e métodos ainda não estão descolonizados e ainda não estão sintonizados às formas que pessoas, passado e presente se relacionam com suas próprias histórias.

Por outro lado, alguns arqueólogos (e outras escolas históricas) têm interpretado encontros coloniais e assentamentos como o momento decisivo nas histórias indígenas, um momento que ou para essas histórias ou as redireciona (veja Hart et al, neste volume ${ }^{3}$ ). Povos Indígenas que passaram por essa porta de entrada fundamental são comumente vistos como significativamente alterados, como uma amalgama de diferentes culturas, desconectados de suas tradições, como novas formas de cultura completas, ou pior como não autênticas. Isso pode ser chamado de short purée - a mistura e esmagamento de culturas Indígenas e coloniais em um pedido relativamente curto. O short purée toma uma forma extrema com o trabalho de niilistas demográficos, mas outras variantes podem ser encontradas nessas abordagens que privilegiam a experiência nova ou criativa do colonialismo - uma espécie de “jogada livre" de símbolos e coisas - ao custo de conhecimentos localizados e experiências de atores sociais Indígenas. Também há um profundo legado nas abordagens aculturais do meio do século XX.

Por outro lado, alguns arqueólogos e poucos antropólogos históricos optam por minimizar o impacto do colonialismo, ou pelo menos o recontextualizam à luz de histórias indígenas de longo prazo que se estendem por séculos, se não por milênios, antes da chegada dos colonizadores Europeus. Independente de suas várias origens teóricas, vou me referir a essa orientação como a longue durée. Essa orientação garante primazia à

\footnotetext{
${ }^{1}$ Traduzido por Raquel Caldas Nolasco, Universidade Federal de Minas Gerais. E-mail: quelcnolasco@gmail.com

${ }^{2}$ University of Massachusetts. E-mail: stephen.silliman@umb.edu.

${ }^{3}$ NT: Hart, S. M.; Oland, M; Frink, L. 2012. Finding transitions: global pathways to decolonizing indigenous histories in Archaeology. In: Hart, S. M.; Oland, M; Frink, L. Decolonizing Indigenous Histories: Exploring "Prehistoric/Colonial" Transitions in Archaeology. Tucson (AZ), University of Arizona Press.
} 
agência, tradição e estruturas culturais indígenas que tanto precedem como rivalizam aquelas dos colonizadores e permitem uma visão da ação Indígena como contribuinte, em parte pela direção da história. Tal visão algumas vezes cai metaforicamente ou conceitualmente na escola de história dos Annales. Outras formas podem ser vistas no famoso trabalho de Marshall Sahlins no Pacífico (Sahlins, 1981, 1985) ou em trabalhos mais recentes com ênfase em mitos e metáforas Indígenas (Vitelli, 2011).

Ambas as perspectivas têm valor, mas também têm limitações no estudo de histórias Indígenas através da “grande-divisão”, também chamada de períodos pré-histórico/histórico ou pré-contato/pós-contato (Ver Scheiber and Mitchell, 2010). Meu objetivo neste capítulo é ressaltar as posições e seus problemas, e propor uma reorientação para a escala de memória e prática (ver Stahl, neste volume ${ }^{4}$ ) como uma maneira potencial de resolver alguns dos problemas levantados por esses polos opositores heurísticos. Essa resolução surge, e pode contribuir com, de abordagens colaborativas descolonizadoras da arqueologia e história. De fato, meu próprio pensamento sobre isso deriva da interseção da teoria pós-colonial, teorias sociais da memória, dados arqueológicos e minha parceria de longa data com a Nação Tribal Pequot Oriental do sudeste de Connecticut para estudar seu passado. Recontarei algumas das interpretações e contextos dessa pesquisa aqui para fazer esses pontos conceituais.

\section{O SHORT PURÉE}

Na perspectiva do short purée, o colonialismo serve como o ponto de inflexão mais proeminente no arco das histórias Indígenas. Em sua forma mais extrema, um modelo de "impacto fatal" propõe que as culturas e pessoas Indígenas eram fundamentalmente alteradas pela presença dos colonizadores Europeus e pelas colônias frequentemente ao ponto de se tornarem irreconhecíveis nos termos de suas formas culturais anteriores. Essa narrativa permanece entranhada na percepção geral do público Americano sobre a história dos Nativos Americanos e merece alguma atenção aqui, apesar de ter desaparecido da maioria das agendas de pesquisa arqueológica. O mecanismo de impacto fatal varia, no entanto. Alguns vêm patógenos introduzidos por Europeus como o maior agente debilitante, contribuindo como fizeram com pandemias de mortalidade significativa. Aqueles passando por esse gargalo são pensados como tendo perdido muito de sua integridade cultural e tendo se transformado em algo novo, particularmente com recombinações de diferentes grupos culturais (Dobyns, 1991; Dunnell, 1991). Tais perspectivas identificam formas de pós-contato - etnohistórico, etnográfico e comunidades Nativas contemporâneas - misturadas e desconectadas de suas histórias de longo prazo.

Outras escolas veem sistemas coloniais de autoridade, proselitismo religioso, reassentamentos forçados, programas aculturativos e a introdução e adoção de nova cultura material como mecanismos do short purée. O último, inicialmente emoldurado como pesquisas de aculturação, tem oferecido um importante ponto de entrada para arqueólogos para medir mudança e continuidade cultural em sociedades Indígenas. Aqueles que seguiram a chamada aculturação frequentemente viram o purée a caminho, quando itens da cultura material de origem Europeia surgiram em contextos culturais de Nativos Americanos. Por outro lado, aqueles que

\footnotetext{
${ }^{4}$ NT: Stahl, Ann B. 2012. When does the history begin? Material continuity and change in West Africa. In: Hart, S. M.; Oland, M; Frink, L. Decolonizing Indigenous Histories: Exploring "Prehistoric/Colonial” Transitions in Archaeology. Tucson (AZ), University of Arizona Press.
} 
estudaram resistência olharam de perto para agentes e práticas subversivas Nativas Americanas que deram curto-circuito no purée. Isso reconheceu o potencial iminente do purée, mas permitiu um mecanismo para desviá-lo.

Ninguém pode negar os severos, e às vezes imediatos, impactos da violência, doença, marginalização, racismo, genocídio e ataques culturais às sociedades Indígenas, mas essas posições analíticas têm limitações e colonizam a historiografia de diversas maneiras. Elas situam histórias Indígenas dentro do colonialismo, ao invés de cruzá-las com ele, dessa forma diminuindo-as. Elas desencorajam a pesquisa arqueológica sobre histórias Indígenas mais recentes, porque privilegiam reinos pré-contato como mais intocados (isto é, mais "Nativos") e mais interessantes (veja crítica em Lightfoot, 1995). Esse é um elemento sutil, mas perigoso - é necessário apenas tabular o número de projetos arqueológicos sobre "primeiros encontros" e "períodos de contato inicial" comparados com estudos sobre os séculos mais adentro do colonialismo e nações de assentamentos. Um foco em períodos anteriores ocorre frequentemente apesar da grande riqueza de informações em arquivos sobre períodos mais tardios, dimensões que poderiam fornecer uma arqueologia histórica mais rica. Além disso, tal ênfase nesses períodos mais antigos separa comunidades Indígenas no presente de seus passados e suas habilidades de exercer reivindicações sobre eles, não apenas pela retórica de impacto fatal, mas também pela prática de não estudar conexões mais recentes em suas cadeias históricas. (Lightfoot, 2006). Essa separação tem enorme importância política em um mundo moldado por desafios para os direitos e histórias Indígenas em uma variedade de nações colonizadas como os Estados Unidos.

Um duplo padrão está em trabalho. Aqueles grupos e indivíduos envolvidos que tomaram decisões difíceis para sobreviver um ataque colonial agora recebem a culpa por suas mudanças pré-assumidas, apesar do fato de o colonialismo criar muito deste contexto com políticas de deslocação, violência e marginalização. Como um purée, esse processo aparece como uma desordem ou diluição ao invés de uma acomodação estratégica que permitiu a sobrevivência cultural. Formas de mudança no contexto de suas próprias e únicas histórias - suas longas durações - muitas vezes passam sem ser reconhecidas. Tais interpretações do colonialismo dão licença aos recém-chegados e desfavorece aqueles que viveram em paisagens particulares por várias gerações. Esses recém-chegados que levaram adiante projetos explicitamente coloniais seguiram em frente no tempo como mais de si mesmos - isso é, os Britânicos se tornam mais Britânicos por causa do colonialismo, mesmo que o papel do colonialismo esteja frequentemente escondido (Johnson, 2006) - enquanto aqueles que normalmente sofriam com esses projetos coloniais se tornam menos de si mesmos, pelo menos de acordo com índices de mudança cultural de longa data utilizados por arqueólogos, antropólogos e outros. Se o colonialismo constitui parte do se tornar nacional para os Britânicos, Franceses, Espanhóis e Anglo-Americanos, por que não pode ser para os Mohawk, os Hopi e os Tlingit também? A resposta: Os Nativos Americanos, conceitualmente, viraram purée, enquanto os colonizadores e seus descendentes permaneceram não misturados, apesar de um número de adições e acomodações resultantes de suas interações - econômica, social, política, pessoal, íntima - com povos Indígenas. Essa conclusão permanece firme na mente do público e repetidamente na de acadêmicos também, apesar das pesquisas extensivas em lugares como La Florida Espanhola que demonstram compartilhamento de elementos culturais entre colonizadores e colonizados em um processo de transaculturação (Deagan, 1998).

Abordagens pós-coloniais recentes têm tentado reexaminar esse short purée com um olhar mais afiado para entender negociações Indígenas dessas novas circunstâncias coloniais de formas novas e criativas. A ênfase na agência social e hibridização contornaram algumas das bordas mais ásperas desse short purée, mas elas tendem a 
minimizar algumas das histórias de longo termo das práticas Indígenas no mundo colonial. Isto é, ações no presente colonial são representadas como maneiras de seguir em frente, ou ser irônico (e.g., o conceito de mimetismo introduzido por Homi Bhabba [1985], ao invés de maneiras de também se conectar para trás. Temo que meu próprio trabalho, encorajando arqueólogos a trazer de volta da terminologia da "cultura de contato" quando tentam interpretar o que de outra maneira seriam contextos bastante coloniais (Silliman, 2005), pode ser mal interpretado como um encorajamento deste tipo de purée dentro de um processo colonial decisivo (Jordan, 2009). O colonialismo no primeiro plano como um (o?) fator crítico da vida Indígena em tempos recentes pode enfatizar transformações de curto prazo, hibridização e o novo à custa do longo prazo, prendendo a prática cultural, mas isto não é necessário se perspectivas multi-escala apropriadas forem aplicadas a este tipo de entrelaçamento (Silliman, 2009; Thomas, 1994).

\section{A Longue DuREÉ}

Os arqueólogos se orgulham de suas habilidades em rastrear mudanças e continuidades culturais através do longo prazo. Essas profundidades temporais extensivas marcam, de fato, uma das contribuições chave da arqueologia ao inquérito histórico e a ciência social. Dos palimpsestos arqueológicos de Binford (Binford, 1981) e histórias ambientais de longo prazo ao engajamento com a Escola de História dos Annales (Knapp, 1992) e os debates sobre perspectivismo de tempo (Bailey, 2007; Holdaway \& Wandsnider, 2008; Murray, 1999), muitos arqueólogos gravitaram rumo à interpretação de tendências diacrônicas profundas nas sociedades humanas, como se fosse uma das únicas disciplinas com o poder para fazê-lo. Os debates foram enfurecidos sobre como certos padrões históricos e culturais poderiam operar fora da percepção e além do ciclo de vida de agentes sociais e humanos, e se o registro arqueológico deveria ser interpretado com escalas temporais diferentes e estruturas teóricas de aquelas normalmente aplicadas às configurações etnográficas. Isso continua sendo uma força da arqueologia.

Muitos relatórios de gestão de recursos culturais nos Estados Unidos oferecem uma variação diferente nesse foco em histórias profundas. Mesmo aqueles relatórios que tratam dos períodos mais recentes e mesmo talvez a arqueologia de sítios não Nativos contêm a obrigatória “seção de plano de fundo" na área "pré-história”, normalmente incluindo tudo desde o mais antigo período Paleoíndio. Essa prática parece ter se desenvolvido a partir de uma abordagem arqueológica à história da terra ao invés de uma história dos processos sociais, culturais, econômicos e políticos que podem ou não ter cruzado com histórias tão antigas. Menciono isso não para sugerir que tais planos de fundos não são importantes ou irrelevantes, já que realmente precisamos apreciar melhor os detalhes da história em lugares e tempos específicos e a presença indígena à longo prazo, mas para perguntar-nos sobre o uso incerto e escorregadio de histórias estendidas por arqueólogos simplesmente porque nós temos a habilidade de acessá-las.

Diversos estudos de histórias Indígenas de longo prazo na América do Norte colonial destacaram o valor de tais visões estendidas como um contraponto ao purée, as histórias de curto-prazo. Kulisheck (2010) identificou maneiras que a mobilidade Pueblo, agregação e padrões de migração à longo prazo definiram o contexto para mudança demográfica no início da colonização Espanhola no Sudoeste Americano. Gallivan (2004, 2007) demonstrou os impactos de quatro séculos de vida Indígena em Virginia Tidewater em respostas particulares de Nativos Americanos à colonização Inglesa no início do século XVII (ver também Hantman, 
1990). Em um estudo marco, Lightfoot e colegas (1998) revelaram como histórias mais aprofundadas sobre Nativos Californianos e Nativos do Alasca guiaram e informaram suas ações em uma colônia Russa no século XIX no norte da Califórnia.

Essas perspectivas não esticam o tamanho da longue durée como fazem alguns "pré-historiadores" nos debates acima mencionados sobre escala e o registro arqueológico, mas eles revelam o poder e promessa de situar histórias Indígenas em suas próprias origens e trajetória. Contudo a fascinação da longue durée deve ser temperada com perspectivas multiescala e uma apreciação por aquilo que acontece nessas escalas (Lightfoot, 1995; Silliman, 2009; Richard, neste volume ${ }^{5}$; Tveskov, 2007). Só porque nós podemos estudar histórias como padrões culturais e espaciais de longo termo através de vários séculos ou milênios, não quer dizer que nós devemos sempre fazer isso. Um estudo diacrônico não é simplesmente levar um estudo de contexto histórico o máximo possível para o passado. A história deve ser narrada, lembrada, corporificada, institucionalizada ou de outra forma transmitida para ter um impacto (Trouillot, 1995). Talvez as pessoas do passado nem soubessem as escalas de história que os arqueólogos podem hoje visionar através do registro material, ou talvez eles soubessem delas, mas escolheram não mobilizar esses recursos de memória enquanto negociavam mudança e continuidade em seus próprios tempos de vida. Essas devem ser estabelecidas em contextos reais em vez de adotá-los de início. Em outras palavras, devemos pelo menos focar o máximo possível na memória social e mecanismos, para recuperação e encorajamento deles ao invés dos nossos. Precisamos usar mais tempo narrativo do que tempo cronológico para conectar nossas interpretações arqueológicas aos engajamentos das pessoas do passado com as histórias que associamos a elas (Lucas, 2005). Além disso, deveríamos evitar a armadilha de assumir que a arqueologia é limitada por grandes varreduras de história na era colonial (e.g., Fagan, 1997:34), quando se pode claramente acessar diferentes núcleos familiares e eventos que podem se estender por uma mera década ou menos.

A história é orientada de maneira diferente, quando enfatizamos um foco pesado ou sem críticas na longue durée. Estender as histórias Indígenas fundo no tempo serve a um objetivo político de situar ainda mais pessoas em suas paisagens e respeitar as possibilidades de suas conexões ancestrais. Muitos, especialmente estudiosos Nativos Americanos e membros das comunidades, apoiariam tais objetivos valiosos, e esses efeitos devem ter o devido peso. Contudo um perigo espreita em versões mais extremas, porque a perspectiva também assume que pessoas Indígenas podem ser mais "tradicionais" e se ligarem aos seus passados e por tanto, serem menos capazes de re-moldurar seus campos sociais, culturais, econômicos, e políticos em casos de risco empírico grave como aqueles trazidos pelo colonialismo. Isto é, suas negociações de memória social podem ser posicionadas de maneira errada dentro do tempo arqueológico ao invés de suas próprias perspectivas de passado. Suas continuidades estratégicas podem ser vistas como mudanças passivas. Nada disso nega o fato de que muitos povos Indígenas têm conexões muito mais seguras com seus passados profundos do que muitos povos não indígenas em nações colonizadas. Isso só acentua a necessidade de saber como essas "conexões" acontecem e reconhecer como elas foram usadas contra pessoas "tradicionais", que fizeram escolhas para "mudar" e foram rotuladas como não sendo mais as mesmase, consequentemente, sem direitos para reivindicar.

Somado a isso, arqueólogos precisam prestar contas pelas diferentes aplicações de histórias antigas. Quão longe no passado nós estendemos essa longue durée, e por que essa extensão parece variar dependendo se os

\footnotetext{
${ }^{5}$ NT: Richard, François G. 2012. Lost in tradition, found in transition: scales of indigenous history in Siin, Senegal. In: Hart, S. M.; Oland, M; Frink, L. Decolonizing Indigenous Histories: Exploring "Prehistoric/Colonial” Transitions in Archaeology. Tucson (AZ), University of Arizona Press.
} 
grupos em questão são Indígenas ou colonizadores? Muitos arqueólogos estariam interessados em pensar como padrões culturais de longo termo, particularmente mentalidades, podem conectar grupos Pueblo do século XVII com Canyon Chaco 500-600 anos antes, ou grupos Caddo no século XVIII no Vale do Mississippi com Cahokia e outras tribos do Mississippi de 500 a 700 anos atrás. Ainda, como muitos iriam seguir regularmente a Inglaterra do século XX como ponto chave para entender padrões culturais coloniais no século XVII em Jamestown ou Plymouth na costa Atlântica dos Estados Unidos? Não estou argumentando que ambas as abordagens estão certas ou erradas, mas, ao contrário, estou comentando sobre suas prevalências como uma função de adequação presumida. Colocado de maneira mais extrema, podemos imaginar mais facilmente arqueólogos pensando que um monte de terra arcaica do Nordeste da Lousiana de 5.000 anos têm alguma relevância para culturas Nativas Americanas encontradas pelos Franceses e Espanhóis no sudeste da América do Norte bem antes deles considerarem o papel do Stonehenge nas colônias culturais britânicas. A escala de tempo absoluta é a mesma, a diferença está nas percepções de mudança e distância cultural.

Talvez essas hipóteses exagerem o caso, mas deixe-me colocar esse ponto sobre escala em um desdobramento mais predominante de problemáticas de escalas históricas de longo termo. Quando pesquisam as dimensões de mudança cultural e continuidade entre sociedades Nativas Americanas na América do Norte, os arqueólogos normalmente formam uma "linha de base etnográfica” para comparação que inclui práticas culturais conhecidas desde o período imediato pré-contato. Essa linha de base pode ser desenvolvida usando observações históricas de primeiro encontro ou pesquisas arqueológicas intensivas, cuidando para não colapsar o tempo com o intuito de criar um único análogo (Stahl, 1993). Essa abordagem permanece altamente válida para acessar pós-contatos imediatos ou mudanças e continuidades culturais iniciais em Nativos Americanos ou outras sociedades Indígenas, como demonstrado em clássicos como Lightfoot et al. (1998). Ainda, por quanto tempo uma linha de base faz sentido para acessar mudança e continuidade cultural? Usamos uma abordagem similar para acessar mudanças e continuidades culturais Européias em cenários coloniais?

A resposta para a questão anterior depende da escala considerada, mas, na prática, arqueólogos tendem a se prender a linha de base para todas as comparações subsequentes. Como resultado, muitos sítios arqueológicos associados a Nativos Americanos na era colonial/pós-contato, se não avaliados criticamente, podem ser facilmente interpretados como representantes de mudança (em contraste com continuidade) quando comparados com linhas de base distantes (ver Silliman, 2009). Esse ponto de comparação não continua no tempo como as culturas ou grupos Indígenas em questão o fazem. No entanto alguns arqueólogos começaram a pressionar essas fronteiras (e.g. Harrison, 2002; Hodge, 2005). Por exemplo, Wagner (2010) demonstra como "nativistas" do Centro-Oeste que queriam proteger seu modo de vida "Indígena" no final dos 1700 e início dos 1800 não enfatizavam conjunto eterno, com milênios de idade de práticas sem mudanças, mas, ao contrário, enfatizavam uma amalgama coerente de práticas culturais que se desenvolveram através de várias gerações como resultado de suas interações com vizinhos Nativos Americanos e Europeus. Abordagens arqueológicas sobre movimentos de revitalização de Nativos Americanos na era colonial também revelaram práticas igualmente criativas, ancoradas e variavelmente temporalizadas (Liebmann, 2008).

Ainda assim, muito trabalho ainda precisa ser feito nessa fronte. No domínio público, quantos cidadãos americanos pensam sobre pessoas indígenas contemporâneas em termos de igualdade, comparado aos seus ancestrais do século XIX ou mesmo século XX, ou em termos de suas diferenças comparadas com os períodos coloniais iniciais? Na Nova Inglaterra de hoje, por exemplo, os Pequot são tipicamente pensados unicamente como a mais poderosa tribo que foi altamente dizimada no início do século XVII na Guerra Pequot pela milícia 
colonial Britânica e seus aliados ou como donos lucrativos financeiramente do Foxwoods Casino e Resort abertos da década de 1990 (mesmo este último se referir apenas aos Pequot Mashantucket e não aos seus primos não reconhecidos no âmbito federal, os Pequot Orientais). Sem uma alteração de linha básica que considere as mudanças e continuidades no final dos séculos XVII, no XVIII e XIX, ou o que é apenas algumas décadas de quase 400 anos desde a Guerra Pequot até o Foxwoods Casino, observadores das culturas e povos Pequot consideram que as pessoas de hoje são radicalmente diferentes das pessoas do "período de contato" de ontem. Academicamente, essas obsessões que historiadores têm com a Guerra Pequot, e outras do século XVII, frequentemente deixam subsequentes eras desprovidas de história e cultura Nativa, como se esses grupos Nativos Americanos não houvessem continuado (mas veja exceções em Den Ouden, 2005; Mandell, 2008; McBride, 1990, 1993, 1996). A lacuna acentua a mudança, simplifica cadeias históricas complexas e serve como receita para comparações de longo-termo problemáticas. Além disso, produz um falso senso de longue durée, um ponto congelado no tempo que obscurece as estratégias de curto-prazo, decisões e processos que conectam o passado ao presente e que dão a padrões culturais de longa duração seu verdadeiro poder.

A resposta para a segunda pergunta - se aplicamos a mesma escala para sociedades não Indígenas - é um fácil não, mas ela traz o mesmo ponto. Os arqueólogos históricos interessados em assentamentos coloniais europeus determinam como sua linha de base o encontro entre os colonizadores e grupos Indígenas bem como os modos de vida pré-encontro (travessia pré-Atlântica) de colonizadores Europeus? Normalmente não. O ponto chave, no entanto é que essa linha de base, mesmo se usada, não permanece fixa nesse encontro. Onde estão os estudos que olham para as transformações do século XVIII Britânicas, Espanholas, Francesas e depois para práticas Euro-americanas criadas contra seus padrões "pré-contato"? Os colonizadores britânicos são julgados como tendo mudado significativamente desde esses períodos, ou são eles considerado como transformações das mesmas pessoas? Para ser contundente, muitas pessoas nos Estados Unidos acham que Nativos Americanos dirigindo carros, comprando cassinos, escutando hip-hop e vivendo em apartamentos urbanos devem certamente serem diferentes de seus ancestrais os quais deviam ter - normalmente imaginados estereotipicamente - andado a cavalo, vestido pele de gamo, lutado contra a Cavalaria Americana e vivido caçando, colhendo ou plantando. Ainda assim, eles não veem as mesmas desconexões entre seus próprios carros, músicas e apartamentos e seus ancestrais do passado, com transporte de cavalo e charrete, perucas brancas, rifles de pederneira e canetas de pena de tinteiro.

Mais pungentemente, Euroamericanos contemporâneos não são questionados sobre as similaridades ou conexões com seus ancestrais nas primeiras décadas da nação Americana no final dos 1700; ainda assim Nativos Americanos raramente ganham o benefício dessa pequena escala de tempo para os cientistas sociais, humanistas, políticos, administradores e membros públicos leigos que avaliam suas mudanças e continuidades. Parte desse desequilíbrio está relacionado a esquemas avaliativos desiguais aplicados a indígenas e colonizadores, e parte está ligada a grandes escolhas e conhecimentos populares sobre as fibras históricas que tecem juntos (e levam adiante) histórias Euro-americanas, devido em grande parte a um excesso de confiança nas fontes escritas quando comparadas às orais e materiais. Tal tecido deixa essas histórias com uma narrativa mais desatada, não pontuada por lacunas que sugeririam grandes interrupções culturais entre esses períodos icônicos e o mundo de hoje. Arqueólogos precisam intervir em todas essas três arenas, mas eles precisam também quebrar algumas das perspectivas que os sustentam. 


\section{EQUILIBRANDO AS BALANÇAS: UM EXEMPLO DA NOVA INGLATERRA}

As dicotomias do short purée e da longue durée foram apresentadas para fazer uma série de apontamentos sobre como nós pensamos sobre tempo, cultura e materialidade. Elas oferecem questões de perspectiva sobre história que devemos escutar, para que nossas interpretações permaneçam sensíveis às experiências vividas no passado e às ramificações políticas de como enquadramos nossas questões. Minha abordagem não foi de reconciliar o short purée e a longue durée como se eles representassem a distinção entre evento versus processos de longo termo, mas, ao contrário, foi de situar o pensar sobre tempo no contexto daqueles que o viveram. Similarmente, não estou defendendo uma espécie de meso-escala de análise como uma solução para esses problemas. Outras abordagens de "mesoescalas" têm se mostrado bastante convincentes (Voss, 2008), mas estas têm focado em mesoescalas de processos e sistemas (tais como trabalho e economia em contextos coloniais), não necessariamente no tempo. Uma escala de médio alcance de análise temporal não é necessariamente mais adequada para todos os contextos coloniais do que uma perspectiva de tempo profundo ou raso, mesmo que seus benefícios sejam revelados no caso abaixo. Isso depende do contexto. Meu argumento é muito menos sobre a escala em que fenômenos sociais, culturais e históricos se manifestam, do que sobre como as pessoas no passado sabiam e mobilizaram histórias e memórias de curto, médio e longo prazo. Ao invés de reconciliar escalas, esta abordagem as reequilibra para coincidirem com as pessoas que viveram e lembraram-se delas.

Essa discussão até agora acentua os problemas de escala encontrados na arqueologia de pessoas Indígenas bem antes, dentro e através do período colonial, particularmente cara a cara com a arqueologia dos colonizadores Europeus. O restante deste capítulo considera como esses problemas podem ser mediados através de cuidadosa atenção às escalas temporais e a materialidade em um caso de estudo específico. Muitos dos detalhes foram apresentados em outro lugar (Silliman, 2009), mas gostaria de desenvolver mais as ideias apresentadas aqui com este estudo empírico.

A reserva dos Pequot Orientais em North Stonington, Connecticut, fornece um cenário ideal para estudar colonialismo e histórias Indígenas, sendo uma das primeiras reservas com ocupação mais longa no que é hoje os Estados Unidos (Figura 1). Concedida em 1683, depois de décadas de colonialismo holandês e britânico incluindo a famosa e infame Guerra Pequot entre 1636-1637 - na Nova Inglaterra Meridional, a propriedade de antes 280 acres, agora de 225 acres, foi quase exclusivamente residência de membros da comunidade dos Pequot Orientais, ambas de longa data e novas através de casamento, com a única infiltração Européia/Euroamericana acontecendo basicamente através da desmontagem de pastos e cercas de fronteiras. Apesar de não ser grande, a reserva permanece notavelmente não perturbada, com floresta secundária, umas poucas trilhas e estradas sujas, com residências ocupadas localizadas no perímetro distante de muitos sítios arqueológicos conhecidos. A reserva também funciona como um espaço cultural e histórico ativo para a Tribo Nacional dos Pequot Orientais, que fornece um local único para pesquisa colaborativa entre arqueólogos e povos Indígenas (Silliman \& Sebastian Dring, 2008). Desde seu início em 2003, o projeto de pesquisa, conhecido como Escola de Campo Arqueológico Pequot Oriental, teve como seu objetivo principal a documentação da variabilidade temporal e espacial das famílias dos Pequot Orientais desde o final do século XVII até a metade do século XIX, usando uma variedade de esforços de mapeamento, sondagens superficiais e sub-superficiais, e escavações em grande escala. 


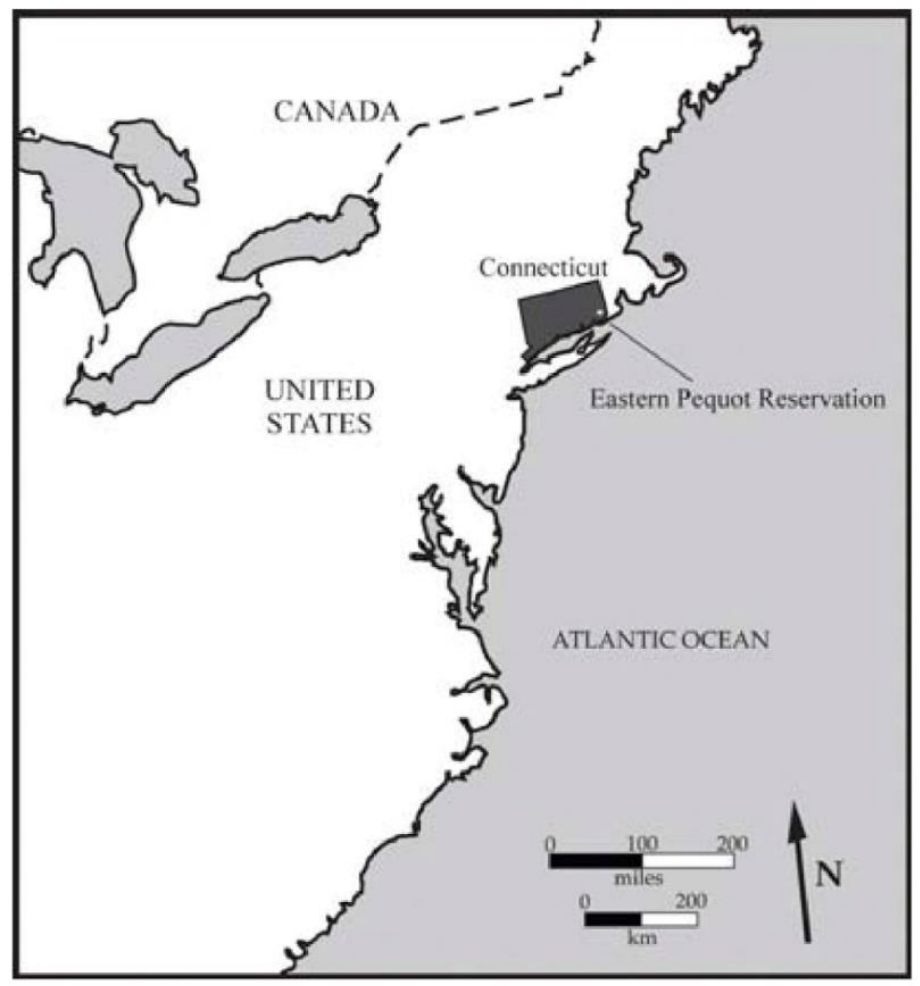

Figura 1: Mapa da América do Norte Oriental mostrando o Estado de Connecticut e a reserva da Tribo Nacional dos Pequot Orientais.

As estratégias de análise combinadas permitiram pesquisar diversos sítios e diversos contextos fora dos que vão do período entre 1740 a 1850 (Cipolla, 2008; Silliman, 2009; Silliman \& Witt, 2010). É suficiente dizer aqui que cinco casas familiares escavadas extensivamente até agora daquele século - duas mais reportadas em Silliman (2009) foram escavadas no verão de 2008 e 2009 - fornecem uma visão de práticas culturais dos Pequot Orientais em terras ancestrais e entre esforços para sobreviver na Nova Inglaterra colonial, mas colocadas em parâmetros arquitetônicos, artefatuais e alimentares marcados em grande medida por materiais e tecnologias derivados de Europeus/Euroamericanos. Isto é, Pequot Orientais residentes representados por essas casas usaram cerâmicas importadas, tais como redware, slipware, creamware, pearlware, grés e porcelana; utilizaram uma variedade de implementos metálicos, como facas, garfos e fechaduras; arquitetura ancorada e talvez mobiliário em madeira com pregos e prendedores de metal; bebiam de garrafas de vidro verde escuro e copos de vidro transparente; vestiam ou produziam roupas com uma variedade de botões de metais; cozinhavam em chaleiras de ferro; comiam e talvez mantinham estoque vivo de vacas, porcos e ovelhas; $\mathrm{e}$ às vezes utilizavam a moeda local. Quatro das cinco casas eram casas com moldura de madeira com pregos e janelas de vidro, e a quinta pode ter sido uma estrutura hibrida entre uma tradicional weetu (ou wigwam) e uma casa emoldurada.

Seria tentador em certos círculos arqueológicos argumentar que cada uma dessas cinco casas - quando comparadas à linha básica pré-contato de caça, coleta, cultivo, coleta de conchas, pesca, fabricação de cerâmica, lascamento de instrumentos de pedra, confecção de contas de concha, mudanças sazonais e viver em vilas weetu - indicam mudança cultural significativa. Alguém pode até dizer que isso mostra um short purée ou uma submersão colonial de práticas tradicionais. Um contador moderado poderia se munir com as descobertas arqueológicas de alguns, apesar de não muitos, materiais líticos e a incorporação da pesca, coleta de conchas e 
caça nas práticas alimentares. Esses poderiam indicar conexões ativas com modos ancestrais. Ou talvez mesmo práticas híbridas poderiam ser mencionadas com o ocasional objeto de vidro lascado ou a pederneira trabalhada bifacialmente, e a presença de contas de vidro em pequenos números (de forma onipresente, no entanto) nos sítios. A descoberta de três instrumentos de pedra provavelmente de alguns milhares de anos que foram guardados em uma casa de Pequot Orientais no século XIX também pode contribuir para aquele contraargumento (Silliman, 2009:221-224). Estes podem levar a um equilíbrio entre o short purée de influxo colonial e a longue durée de modos ancestrais Nativos. Apesar da possível força desse contra-golpe, o argumento ainda conta com a linha básica estática para comparação e com identificadores culturais atuais de objetos materiais, o último dos quais são abordados de forma mais completa em outro lugar (Silliman, 2009). Eles cairiam diretamente nas noções de tempo, cultura e materialidade ainda colonizadas.

Em contraste, os padrões arqueológicos poderiam ser dimensionados para o século desses membros da casa vivendo na reserva. Com esse ponto de vista analítico, a cultura material sugere uma forte continuidade em diferentes famílias em lugares diferentes na reserva para esses 100 anos. Essa perspectiva ressalta as formas como práticas familiares e memórias individuais podem ter mantido comunidade e bem-estar através de novos materiais, tecnologias e comidas. Ela liberta aqueles indivíduos Pequot Orientais particulares no passado que tiveram que se basear em suas "bases pré-contato" nos nossos termos para permanecer Nativos Americanos ou sobreviver culturalmente. Ela permite a possibilidade de membros da comunidade dos Pequot Orientais basearem-se variavelmente em seus passados de acordo com idade, gênero, posição econômica e outros fatores ao invés de assumir que todos Nativos Americanos nessa comunidade (ou em qualquer outra) se baseiam no mesmo pacote de conhecimentos, práticas e memórias que nós esperamos que eles tenham - isto é, o "passado tradicional" - independente da passagem do tempo.

Similarmente, isso garante uma espécie de autenticidade na ação para aqueles residentes no início do século XIX que se basearam em ações ancestrais recentes do final do século XVIII e que garantiram um domínio na reserva em momentos de dificuldade política e econômica, ao invés de culpá-los pelo crescente distanciamento de seus antecedentes pré-contato de 200 anos antes. Finalmente, essa perspectiva reconhece que tecnologias e materiais derivados dos Europeus podem ter se tornado componentes de práticas domésticas e talvez até tradições familiares ou da comunidade. Gerações sucessivas de Pequot Orientais (ou outros Nativos Americanos) não tiveram cada um de seus pais adotando bens de mercado como implementos cerâmicos e metálicos. Eles estavam usando-os. Um foco nas linhas de base etnográficas imóveis esquece esse aspecto, avaliando como fazemos com frequência cada instância da cultura material "Européia” ou "Nativa” nos sítios, abrangendo gerações como se elas fossem o resultado direto de uma escolha imediata, consciente e constante entre novo-antigo, colonizador-colonizado ou Europeus-Indígenas. Tais dicotomias começam a se revelar imediatamente depois o chamado contato entre Europeus e povos Indígenas (ver também Hart, neste volume ${ }^{6}$; Rodríguez-Alegría, neste volume ${ }^{7}$ ).

Essa nova perspectiva surge de uma consideração teórica e empírica de escala temporal e ação cultural. Teoricamente, isso garante que arqueólogos não errem muito no lado do short purée. Isso pode tornar os Pequot

\footnotetext{
${ }^{6}$ NT: Hart, Siobhan M. 2012. Decolonizing through heritage work in the Pocumtuck homeland of Northeastern North America. In: Hart, S. M.; Oland, M; Frink, L. Decolonizing Indigenous Histories: Exploring "Prehistoric/Colonial” Transitions in Archaeology. Tucson (AZ), University of Arizona Press.

7 NT: Rodríguez-Alegría, Enrique. 2012. The discovery and decolonization of Xaltocan, Mexico. In: Hart, S. M.; Oland, M; Frink, L. Decolonizing Indigenous Histories: Exploring "Prehistoric/Colonial” Transitions in Archaeology. Tucson (AZ), University of Arizona Press.
} 
Orientais simplesmente consumidores de mercado junto com outros assentamentos e grupos prioritários que buscavam expressar a sua própria agência e construção de significado no contexto capitalista, sem nenhum reconhecimento de suas histórias aterradas e defendidas naquela reserva por 100-200 anos, um lugar que também ficava na paisagem ancestral da maioria dos moradores, bem antes da chegada dos Europeus. Dita perspectiva também assegura que arqueólogos não escorreguem tão facilmente para dentro da longa duração em uma pesquisa por apenas práticas de milênios de idade como teste decisivo para ação social. Uma falta de citação social a esses modos mais antigos de práticas diárias por Pequot Orientais no século XVII e XIX indica não uma falta de consciência desses passados importantes e tradicionais, mas uma convocação estratégica e habitual desses de certos outros passados na vida diária. Algumas vezes isto envolvia vasilhas de redware que os pais utilizaram, enquanto em outros momentos envolvia adicionar ferramentas líticas produzidas milênios antes de uma família do século XIX.

Colocado de maneira diferente, a abordagem foca em uma mesoescala temporal para negociar as oposições de histórias de curto e longo termo. Argumentei anteriormente que a mesoescala de tempo não oferece uma panacéia para os dilemas na arqueologia do colonialismo, mas nesse caso certamente revela aspectos importantes. Isso é feito através do reposicionamento de interpretações para alinhá-las mais com a escala da família e memórias geracionais, as quais frequentemente são os recursos sociais mais próximos sobre os quais os indivíduos se baseiam (e.g., Tveskov, 2007). Arqueólogos têm negligenciado essa escala temporal, mesmo quando eles consideram o papel da arqueologia familiar há décadas. Mais importante, no entanto, essas não representam apenas posições teóricas baseadas em esforços teóricos removidos há algum tempo da arqueologia de campo. Elas são desenvolvidas através de engajamentos com dados empíricos de sítios escavados que encaixam confortavelmente na mesoescala, esticando os indivíduos por duas ou três décadas e coletivamente por mais de 100 anos.

Igualmente relevante é o desenvolvimento dessas ideias em espaços colaborativos de arqueologia Indígena (ver Hart, neste volume ${ }^{8}$ ). Aprendi muito com meus amigos, estudantes, colegas e conselheiros Pequot Orientais durante o andamento desse projeto, e esse aprendizado abriu meus olhos para os desafios e histórias sobre “ser Nativo Americano" na Nova Inglaterra em geral e nos casos específicos de Pequot Orientais, seja em 1700, 1800, 1900 ou 2000. Isso me ajudou a entender a natureza dos ataques públicos oficiais na história da comunidade e na sua integridade cultural e a contribuição infeliz dos antropólogos para parte deste problema com estruturas conceituais fora de moda. Isso me forçou a começar a contabilizar como rastreamos as longas fibras da história que conectam comunidades do presente com seus passados ancestrais. Essa contabilização leva a um entendimento profundo de que esses “comos” ainda merecem nossos esforços de descolonização nas frentes conceituais, metodológicas, práticas, terminológicas e políticas.

\section{CONCLUSÃO}

Os Nativos Americanos nos séculos seguintes aos assentamentos coloniais realizaram uma variedade de conexões com o passado. Terras, materiais, casas e histórias, tudo serviu como repositórios históricos e

\footnotetext{
8 NT: Hart, Siobhan M. 2012. Decolonizing through heritage work in the Pocumtuck homeland of Northeastern North America. In: Hart, S. M.; Oland, M; Frink, L. Decolonizing Indigenous Histories: Exploring "Prehistoric/Colonial” Transitions in Archaeology. Tucson (AZ), University of Arizona Press.
} 
contemporâneos de memória social e materialidades culturais, sob as quais membros da comunidade podiam se basear em suas práticas diárias. Algumas vezes, indivíduos se baseiam nas práticas dos seus pais, que estruturaram suas infâncias; às vezes eles se voltam para seus avôs, com suas histórias e ensinamentos; e outras vezes, eles se baseiam naquelas memórias sedimentadas na paisagem por séculos através de histórias orais, ambiente construídos, ou outros objetos materiais que arqueólogos sabem que existiram no solo em que eles lavraram, cavaram e construíram suas casas. Assim sendo, não devemos considerar esses objetos de origem Européia ou materiais no dia-a-dia de Nativos Americanos como simples parte de um purée cultural, apesar de suas aparências recentes, nem devemos assumir que todas as práticas que esses objetos (ou cultura material mais "tradicional" e "local”) apoiaram foram sempre (ou deveriam ser) ancoradas na longa duração. Essas são questões contextuais a serem respondidas e não elementos a serem assumidos. Todos esses elementos materiais compreendem estratégias conscientes e inconscientes, mentais e corporais, ativas e subjugadas, olhando para frente e pra trás de sobrevivência cultural. Sobrevivência "é mais que sobreviver, mais que perdurar ou meramente responder.... [S]obreviver é uma repudio a dominação, tragédia e vítima.” (Vizenor, 1998;15; ver Silliman n.d.).

Ao permitir uma fluidez na escala temporal na análise arqueológica e ao investigar as formas que agentes sociais fizeram história em suas práticas culturais na vida diária, nós podemos equilibrar cuidadosamente as promessas e problemas da longue durée e do short purée. Às vezes, uma meso-escala de tempo pode servir como ponte conceitual e temporal para resolver essas dicotomias, e outras vezes nós precisamos apenas prestar atenção aos usos das próprias pessoas de tempo e história em suas ações do passado. Focando em memórias sociais e práticas matérias, destacamos os legados coloniais problemáticos em nossas taxonomias acadêmicas e públicas sobre Nativos Americanos-Europeus, mudança-continuidade, pré-história-história e passado e presente, e sugerimos maneiras diferenciadas de interpretar o passado e trabalhar com essas comunidades que descendem e valorizam o mesmo.

\section{AgRADECIMENTOS}

Gostaria de agradecer a Siobhan, Maxine e Liam, por me convidarem para participar neste volume que segue um bem-sucedido simpósio. Também gostaria de agradecer aos vários membros da Tribo Nacional dos Pequot Orientais, que trabalharam comigo neste projeto e apoiaram a pesquisa. Este material é baseado num trabalho apoiado pela National Science Foundation sob o N. 0623532. 


\section{REFERÊNCIAS BIBLIOGRÁFICAS}

BAILEY, Geoffrey N. 2007 Time Perspectives, Palimpsests, and the Archaeology of Time. Journal of Anthropological Archaeology 26:198-223.

BHABHA, Homi 1985 Signs Taken for Wonders: Questions of Ambivalence and Authority under a Tree outside Delhi, May 1817. Critical Inquiry 12:144-165.

BINFORD, Lewis R. 1981 Behavioural Archaeology and the "Pompeii Premise." Journal of Anthropological Research 37:195-208.

CIPOLLA, Craig N. 2008 Signs of Identity, Signs of Memory. Archaeological Dialogues 15 (2):196-215.

DEAGAN, Kathleen. 1998 Transculturation and Spanish American Ethnogenesis: The Archaeological Legacy of the Quincentenary. In Studies in Culture Contact: Interaction, Culture Change, and Archaeology. James G. Cusick, ed. Pp. 23-43. Center for Archaeological Investigations, Occasional Paper No. 25. Carbondale: Southern Illinois University.

DEN OUDEN, Amy E. 2005 Beyond Conquers: Native Peoples and the Struggle for History in New England. Lincoln: University of Nebraska Press.

DOBYNS, Henry F. 1991 New Native World: Links between Demographic and Culrural Changes. In Columbian Consequences, vol. 3: The Spanish Borderlands in Pan-American Perspective. David Hurst Thomas, ed. Pp. 541 560. Washingron, DC: Smithsonian Institution Press.

DUNNELL, Roberr C. 1991 Methodological Impacts of Catastrophic Depopulation on American Archaeology and Ethnology. In Columbian Consequences, vol. 3: The Spanish Borderlands in Pan-American Perspective. David Hurst Thomas, ed. Pp. 561-580. Washington, DC: Smithsonian Institution Press.

FAGAN, Brian. 1997 Clash of Cultures, 2nd edition. Walnut Creek, CA: Alta Mira Press.

GALliVAN, Martin D. 2004 Reconnecting the Contact Period and Late Prehistory: Household and Community Dynamics in the James River Basin. In Indian and European Contact in Context: The Mid-Atlantic Region. Dennis R. Blanton and Julia A. King, eds.Pp. 22-46. Gainesville: University Press of Florida.

GALliVAN, Martin D. 2007 Powhatan's Werowocomoco: Constructing Place, Polity, and Personhood in the Chesapeake, CE 1200-CE 1609. American Anthropologist 109(1):85-100.

HANTMAN, Jeffrey L. 1990 Between Powhatan and Quirank: Reconstructing Monacan Culture and History in the Context of Jamestown. American Anthropologist 92(3):676-690.

HARRISON, Rodney 2002 Australia's Iron Age: Aboriginal Post-Contact Metal Artifacts from Old Lamboo Station, Southeast Kimberley, Western Australia, Australasian Historical Archaeology 20:67-76.

HODGE, Christina J. 2005 Faith and Practice at an Ear1y-Eighteenth-Century Wampanoag Burial Ground: The Waldo Farm Sire in Dartmouth, Massachusetts. Historical Archaeology 39(4):73-94.

HOLDAWAY, Simon, and LuAnn Wandsnider, eds. 2008 Time in Archaeology: Time Perspectivism Revisited. Salt Lake City: University of Utah Press.

JOHNSON, Matthew. 2006 The Tide Reversed: Prospects and Potentials for an Historical Archaeology of Europe. In Historical Archaeology. Martin Hall and Stephen W. Silliman, eds. Pp. 313-331. Malden, MA: Blackwell. 
JORDAN, Kurt A. 2009 Colonies, Colonialism, and Cultural Entanglement: The Archaeology of Post-columbian Intercultural Relations. In International Handbook of Historical Archaeology. Teresita Majewski and David Gaimster, eds. Pp. 31-49. New York: Springer.

KNAPP, A. Bernard, ed. 1992 Archaeology, Annales and Ethnohistory. Cambridge: Cambridge University Press.

KULISHECK, Jeremy. 2010 "Like Butterflies on a Mounting Board": Pueblo Mobility and Demography before 1825. In Across the Great Divide: Continuity and Change in Native North American Societies, A.D. 1400-1900. Laura L. Scheiber and Mark D. Mitchell, eds. Pp. 174-191. Amerind Studies in Archaeology 4. Tucson: University of Arizona Press.

LIEBMANN, Matthew. 2008 The Innovative Materiality of Revitalization Movements: Lessons from the Pueblo Revolt of 1680. American Anthropologist 110(3):360-372.

LIGHTFOOT, Kent G. 1995 Culture Contact Studies: Redefining the Relationship between Prehistoric and Historical Archaeology. American Antiquity 60:199-217.

LIGHTFOOT, Kent G. 2006. Missions, Gold, Furs, and Manifest Destiny: Rethinking an Archaeology of Colonialism for Western North America. In Historical Archaeology. Martin Hall and Stephen W. Silliman, eds. Pp. 272-292. Malden, MA: Blackwell.

LIGHTFOOT, Kent G., Antoinette Martinez, and Ann M. Schiff. 1998 Daily Practice and Material Culture in Pluralistic Social Settings: An Archaeological Study of Culture Change and Persistence from Fort Ross, California. American Antiquity 63(2):199-222.

LOWENTHAL, David. 1996 Possessed by the Past: The Heritage Crusade and the Spoils of History. New York: Free Press.

LUCAS, Gavin. 2005 The Archaeology of Time. London: Routledge.

MANDELL, Daniel. 2008 Tribe, Race, History: Native Americans in Southern New England, 1780-1880. Baltimore: Johns Hopkins University Press.

MCBRIDE, Kevin A. 1990 The Historical Archaeology of the Mashantucket Pequot. In The Pequots: The Fall and Rise of an American Indian Nation. Laurence Hauptman and James Wherry, eds. Pp. 96-I I6. Norman: University of Oklahoma Press.

MCBRIDE, Kevin A. 1993. "Ancient \& Crazie": Pequot Lifeways during the Historic Period. In Algonkians of New England: Past and Present. Peter Benes, ed. Pp. 63-75. Annual Proceedings of the 1991 Dublin Folklife Seminar, Boston University.

MCBRIDE, Kevin A. 1996. The legacy of Robin Cassacinamon: Mashantucket leadership in the Historic Period. In Northeastern Indian Lives, 1632-1816. Robert Grumet, ed. Pp. 74-93. Amherst: University of Massachusetts Press.

MURRAY, Tim. 1999 A Return to the "Pompeii Premise." In Time and Archaeology. Tim Murray, ed. Pp. 8-27. London: Routledge.

SAHLINS, Marshall. 1981. Historical Metaphors and Mythical Realities: Structure in the Early History of the Sandwich Islands Kingdom. Ann Arbor: University of Michigan Press.

SAHLINS, Marshall. 1985. Islands of History. Chicago: University of Chicago Press.

SCHEIBER, Laura L., and Mark D. Mitchell, eds. 2010 Across the Great Divide: Conrinuity and Change in Native North American Societies, A.D. I400-1900. Amerind Studies in Archaeology 4. Tucson: University of Arizona Press. 
SILLIMAN, Stephen W. 2005.Culture Contact or Colonialism? Challenges in the Archaeology of Native North America. American Antiquity 70:55-74.

SILLIMAN, Stephen W. 2009. Change and Continuity, Practice and Memory: Native American Persistence in Colonial New England. American Antiquity 74(2):211-230.

SILLIMAN, Stephen W. n.d. Archaeologies of Survivance and Residence: Reflections on the Historical Archaeology of Indigenous People. In The Archaeology of the Colonized and Its Contribution to Global Archaeological Theory. Neal Ferris, Rodney Harrison, and Michael Wilcox, eds. Oxford: Oxford University Press, in review.

SILLIMAN, Stephen W., and Katherine H. Sebastian Dring.2008. Working on Pasts for Futures: Eastern Pequot Field School Archaeology in Connecticut. In Collaborating at the Trowel's Edge: Teaching and learning in Indigenous Archaeology. Stephen W. Silliman, ed. Pp. 67-87. Amerind Studies in Archaeology 2. Tucson: University of Arizona Press.

SILLIMAN, Stephen W., and Thomas A. Witt. 2010. The Complexities of Consumption: Eastern Pequot Cultural Economics 18th-Century Colonial New England. Historical Archaeology 44(4)46-68.

STAHL, Ann B. 1993. Concepts of Time and Approaches to Analogical Reasoning in Historical Perspective. American Antiquity 58(2):235-260.

THOMAS, Nicholas. 1994. Colonialism's Culture: Anthropology, Travel, and Government. Princeton: Princeton University Press.

TROUILLOT, Michel-Rolph. 1995 Silencing the Past: Power and the Production of History. Boston: Beacon Press.

TVESKOV, Mark. 2007 Social Identity and Culture Change on the Southern Northwest Coast. American Anthropologist 109(3): 431-441.

VITELLI, Giovanna. 2011. Change and Continuity, Practice and Memory: A Response to Stephen Silliman. American Antiquity 76(1):177-189.

VIZENOR, Gerald. 1998 Fugitive Poses: Native American Indian Scenes of Absence and Presence. Lincoln: University of Nebraska Press.

VOSS, Barbara L. 2008. Gender, Race, and labor in the Archaeology of the Spanish-Colonial Americas. Current Anthropology 49(5):861-893.

WAGNER, Mark J. 2010. A Prophet Has Arisen: The Archaeology of Nativism among the Nineteenth Century Algonquin Peoples of Illinois. In Across the Great Divide: Continuity and Change in Native North American Societies, A.D. 1400-1900. Laura S. Scheiber and Mark Mitchell, eds. Pp. 107-127. Amerind Studies in Archaeology 4· Tucson: University of Arizona Press. 